\title{
Asymmetry in the Subsequent Movements' Proportions of Share Prices Included in the WIG
}

\author{
A. SzMagliński
}

Cracow University of Technology, Institute of Physics, Podchorążych 1, 30-084 Kraków, Poland

\begin{abstract}
The intraday data of stock prices allow us to collect in the form of histogram the subsequent movements' proportions in price and time. Here we continue the previous work [ Acta Phys. Pol. A 123, 621 (2013)], concerning the properties of subsequent price movements' proportions in the opposite directions and proportions of subsequent price movements in the same direction. Here we distinguish between the proportions with growing and decreasing second price movement in the proportion. We investigate quantitatively the effect of breaking the turning point of resistance and support levels depending on the percentage size of price movements. In the same way we treat the main peak in the histogram for the equal subsequent price movements.
\end{abstract}

DOI: 10.12693/APhysPolA.127.A-136

PACS: 05.45.Df, 05.45.Tp, 89.65.Gh

\section{Introduction}

The game with the probability of price movements in different directions is the main application of financial markets' technical analysis. In this work we will focus our investigation on widely known as the strongest effect predicted by the technical analysis which is the breaking of support or resistance levels. Here we continue the previous work [1], where the method of analysis the subsequent movements' proportions of prices was introduced.

To perform this analysis we should prepare in some way the transaction's tick data. The idea of how to simplify the price movements is based on the Point \& Figure charts as described in [2] and Kagi charts, which was developed in Japan [3]. The price movements in the opposite directions, which are less than the fixed percentage (called here Minimum Size of Correction - MSC) are ignored. So finally we have price-time series composed of turning points. A great number of subsequent movements' proportions allow us to set them into the histograms. Here we have two kinds of proportions as shown in Fig. 1.

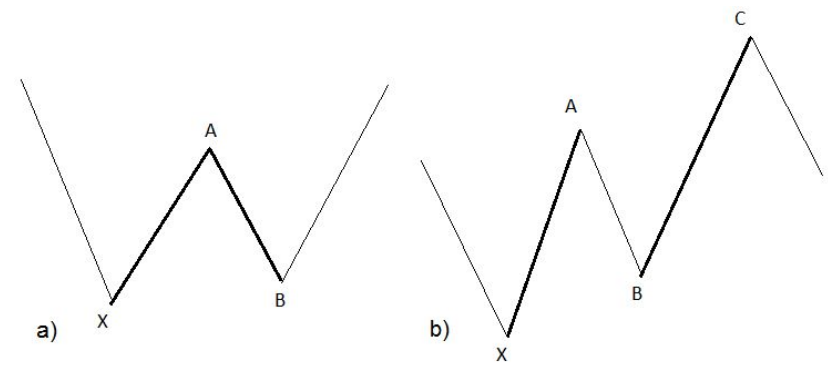

Fig. 1. Two kinds of measuring the price movement proportions in price and time. The first from the left side (denoted by (a)) is the proportion of subsequent movements in the opposite directions and the second (denoted by (b)) proportion of subsequent movements in the same direction.
Additionally we introduce three conditions, so as to avoid price movements corresponding to random fluctuations (for example realization of best buy and sell offers). When share price is less than 1 PLN, every percent of MSC corresponds to $0.01 \mathrm{PLN}$. The second condition is $\mathrm{MSC}>0.50 \mathrm{PLN}$. The third condition states, that every price movement should be composed of more than ten transactions. The calculations have been performed on the intraday tick data since September 1, 2006 when the Warsaw Stock Exchange introduced new quotation steps for the shares until May 9, 2014.

\section{The proportions of subsequent price movements in the opposite directions}

The most simple case corresponds to the subsequent price movements in both directions (Fig. 1a). The proportions of the subsequent movements are set in histogram. Every case of such a proportion is placed in a narrow range of thickness 0.01 , forming a part of the histogram. The same procedure is used for the duration of these price movements. There are shown two histograms for price proportions in Fig. 2. We have distinguished here between the second price movements in proportions which are growing $(A B>0)$ and decreasing ones $(A B<0)$. These histograms are effectively described by gamma probability distribution functions:

$$
\Gamma_{\nu, \lambda}(x)=C x^{\nu-1} e^{-\lambda x} .
$$

In the histogram of price proportions there is sudden drop in probability distribution, corresponding to the breaking of the nearest turning point resistance. The expectations of market participants change after breaking of the resistance. The probability of price movement completion rapidly decreases when the second price movement becomes greater than the first one. This decrease is estimated by fitting two gamma probability distribution functions for $A B / X A$ proportion values: one greater than 1 and the second lesser than 1 . The ratio of transition from one probability distribution to the other is 


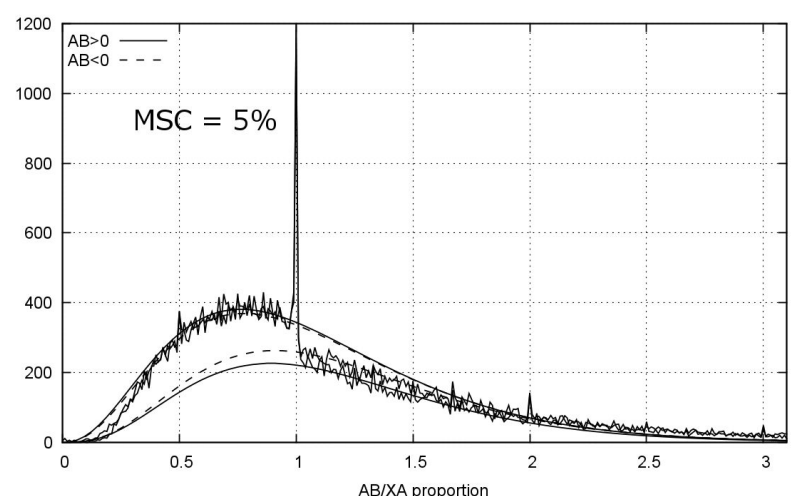

Fig. 2. Two histograms for price proportions with gamma probability distribution functions have been fitted. The histogram for the second price movement growing $(A B>0)$ has been fitted by gamma functions denoted by solid lines and for the second price movement decreasing $(A B<0)$ gamma functions are denoted by dashed lines. In the histogram at value 1 of $A B / X A$ proportion we have sudden drop in probability of completing price movement. This effect is stronger in the histogram for proportions with growing the second price movement.

stronger in general for the lesser magnitude of price movements. The effect of resistance breaking is widely known among the investors and the change in probability of price movement's continuation in the same direction here is given quantitatively. Prior resistance becomes the support area and this can be used as one of possible investment strategy components. One can expect the continuation of price movement. When the increase in price is expected, the strategy gives a buy signal with stop-loss below the support area (for instance a few transaction units below the broken turning point). We can see the asymmetry in the drop of completing price movement probability at value 1 of $A B / X A$ proportion. The change in probability is much stronger for the second price movement growing in proportion $(A B>0)$. This effect is valid for all price scales (Fig. 3). The asymmetry might be explained in a natural way by price limitations. The lower limit is precisely specified as zero. The upper limit is boundless. The natural way to describe prices is a logarithmic scale [4]. The next part of this work should be repetition of the calculations for the logarithmized prices and compare the results for growing and decreasing the second price movements in proportions.

For not a very large price scales (for $3 \%<M S C<12 \%$ ) the effect of resistance breaking $(A B>0)$ is so strong, that changes probability of the price movement completion by $30-35 \%$. This effect is widely known among the capital market investors and very often constitutes the main part of investment strategies. This work gives the quantitative description of this effect depending on the price scale and the price movement direction.

For some values of price and time proportions there are peaks (see [1]). They correspond to proportions in

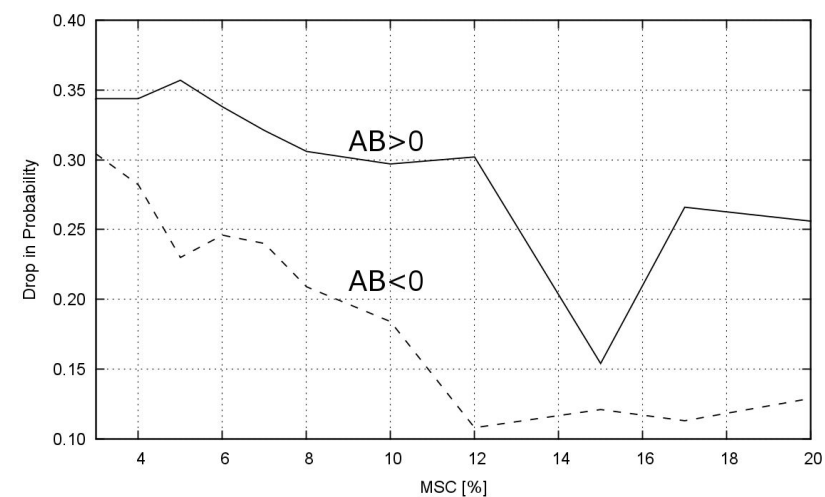

Fig. 3. The effect of support breaking (denoted by dashed line) and resistance breaking (denoted by solid line). Here we have the ratio of transition from one probability distribution to the other depending on the magnitude of price movement. One can see strong asymmetry between these two effects in all price scales.

price and time with greater probability of setting a turning point. The special proportion is the equality of price movements in both directions. This proportion occurs much often than the neighboring proportions. The probability of changing the trend is much greater than in any other nearing price (for the price histogram) or time (in regard to the time histogram [1]). The peaks has its investment application as an accessory condition. The effect intensifies with the overlapping peaks originating from the comparison with other turning points of different price scales.

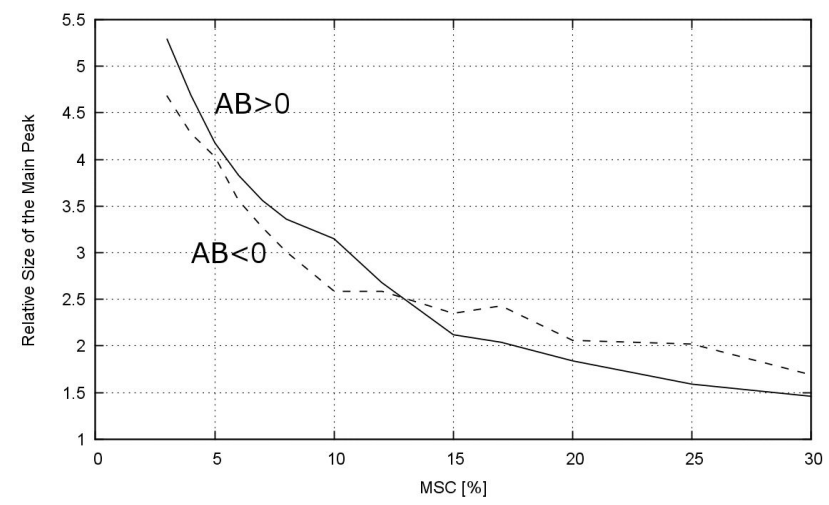

Fig. 4. The relative value of the main peak $(A B=$ $X A)$ in comparison with the neighboring price movements proportions in both directions. In this peak both price movements have the equal values with accuracy of $0.5 \%$.

The relative values of the main peak at value 1 for the price movements' $A B / X A$ proportions (like in Fig. 2), which are the same in value with accuracy of $0.5 \%$, are compared in dependence on MSC at Fig. 4. The relative values of the main peak in comparison with the neighboring proportions relatively quickly decreases with growing 
price scale. For smaller price scales $(M S C<13 \%)$ the subsequent equal price movements for growing the second price movement in the $A B / X A$ proportion occurs more often than for decreasing the second price movements. For larger price scales we have the opposite situation.

\section{The proportions of subsequent price movements in the same direction}

The proportions of subsequent price movements in the same direction (Fig. 1b) have similar properties as the proportions considered in previous section. However, both considered effects are weaker. In case of growing price movements the drop in probability effect is very weak (from 5-15\%) and have no clear dependence on the price scale (Fig. 5 ). In case of decreasing price movements

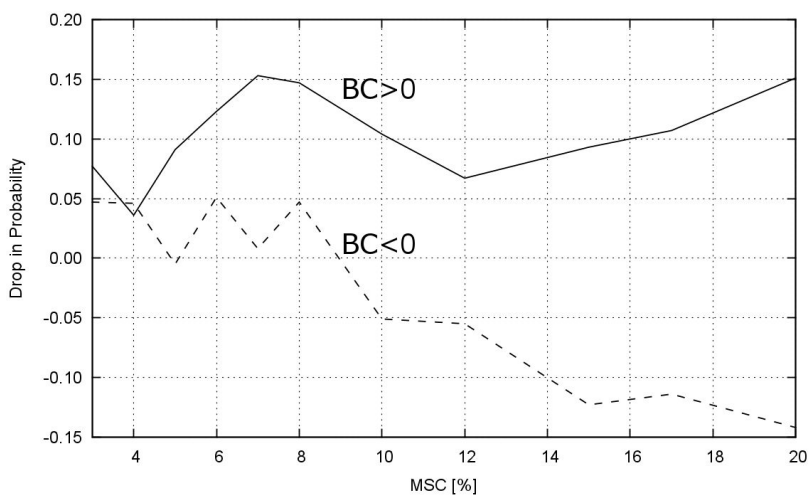

Fig. 5. The ratio of transition from one probability distribution to the other, depending on the price movement magnitude for subsequent price movements in the same direction.

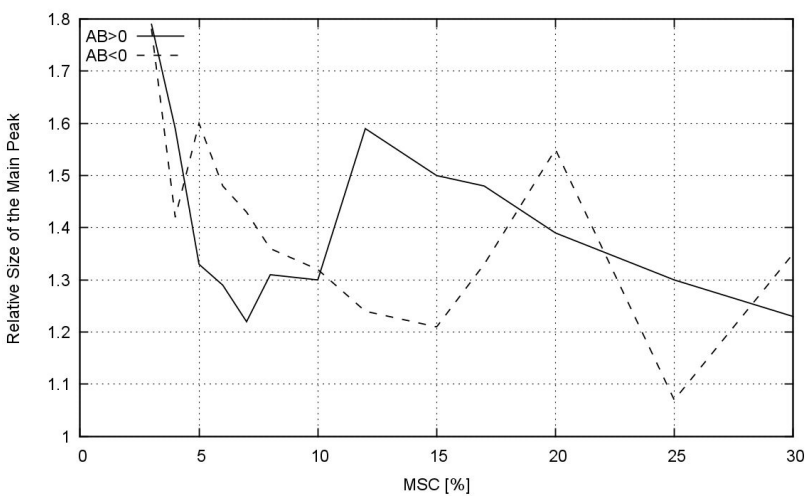

Fig. 6. The relative value of the main peak $(B C=$ $X A)$ in comparison with the neighboring price movements proportions in the same direction. In this peak both price movements have the equal values with accuracy of $0.5 \%$.

the drop in probability is also weak, but is decreasing for the larger price scales and very quickly reaches negative values. It means for larger price scales, the probability of completion the second decreasing price movement just after crossing value 1 of $B C / X A$ proportion, is greater than just before crossing the value 1 .
The value of the main peak for the subsequent price movements in the same direction is rather small (Fig. 6). From this reason its value significantly depends on the background fluctuations.

\section{Conclusions}

In this paper we have demonstrated that the shares have a tendency of price movements' repeatability. For this repeatability is responsible the peak at value 1 in the histograms of $A B / X A$ amd $B C / X A$ proportions. This effect is given quantitatively and is stronger for smaller price scales and subsequent price movements in different directions. The effect of breaking the turning point support and resistance is presented quantitatively. For not so large price scales the resistance breaking makes huge loss of one-third of the price movement completion probability. The greater scale of price movement the lesser decreasing of the probability.

\section{References}

[1] A. Szmagliński, Acta Phys. Pol. A 123, 621 (2013).

[2] Thomas J. Dorsey, Point and Figure Charting. The Essential Application for Forecasting and Tracking Market Prices, John Wiley \& Sons, Inc., 1995.

[3] Steve Nison, Beyond Candlesticks. New Japanese Charting Techniques Revealed, John Wiley \& Sons, Inc., 1994.

[4] P. Samuelson, Economics: The Original 1948 Edition, McGraw-Hill/Irwin. 